\title{
Zinkoxid als photostabiler Luminophor zur optischen Sauerstoffdetektion
}

\author{
Michael Poeplau ${ }^{1}$, Stephan Ester ${ }^{1}$, Bernd Henning ${ }^{2}$, Thorsten Wagner ${ }^{3}$ \\ ${ }^{1}$ Wöhler Technik GmbH, Wöhler-Platz 1, 33181 Bad Wünnenberg, E-Mail: m.poeplau@woehler.de \\ ${ }^{2}$ Elektrische Messtechnik, Universität Paderborn, Warburger Straße 100, 33098 Paderborn \\ ${ }^{3}$ Department Chemie, Universität Paderborn, Warburger Straße 100, 33098 Paderborn
}

\section{Zusammenfassung}

In der vorgestellten Arbeit untersuchen wir Zinkoxid als ein photostabiler Luminophor zur Sauerstoffdetektion. Bisher verfügbare organische Luminophore, üblicherweise Metallkomplexe, weisen gute Eigenschaften bezüglich Sensitivität und Selektivität auf, altern jedoch im Betrieb durch die optische Anregung (Photobleichung). Zinkoxid hingegen ist bekannt als langzeitstabiles lumineszierendes Material und wird unter anderem in Leuchtmitteln verwendet (Bspw. in Leuchtdioden). Wir zeigen die Gasabhängigkeit der Zinkoxid-Lumineszenz mit einer eigens konzipierten Messzelle. Zusätzlich wurden durch simultane Messungen der Lumineszenzemission und des Leitwerts Hinweise auf den Lumineszenz-Quenching-Mechanismus gefunden. Dieser unterscheidet sich qualitativ von den Quenching-Mechanismen an Metallkomplexen.

Keywords: Gassensor, Zinkoxid, Lumineszenz, Luminophor, Photobleichung

\section{Einleitung}

Lumineszenzbasierte Gassensorik, z.B. für Sauerstoff $\left(\mathrm{O}_{2}\right)$, besitzt hervorragende Eigenschaften hinsichtlich Empfindlichkeit und Reproduzierbarkeit. Zusätzlich weisen die als Luminophore verwendeten Metallkomplexe, z.B. basierend auf Ruthenium, eine hohe, inhärente Selektivität auf. Nach Bestrahlung mit kurzwelligem ultraviolettem oder blauem Licht relaxieren die angeregten Elektronen über strahlende Übergänge unter Abgabe einer molekülspezifischen Lumineszenz. Die Reaktion mit Sauerstoff ändert die Molekülorbitale des Luminophors, so dass nun nichtstrahlende Übergänge bevorzugt werden; die Intensität der Lumineszenz nimmt ab (Lumineszenz-Quenching). Die Lumineszenzintensität bzw. deren Abklingverhalten wird als gasabhängige Messgröße ausgewertet [1] und erlaubt so einen einfachen Messaufbau mit kostengünstigen optischen Komponenten [2]. Nachteilig ist jedoch die geringe Stabilität der Metallkomplexe gegenüber kurzwelliger Strahlung. Auf Grund der sogenannten Photobleichung, d.h. die Zersetzung der Moleküle unter Beleuchtung, eignen sie sich daher bisher nicht für kontinuierliche Messaufgaben und werden üblicherweise nur für punktuelle Messungen, beispielsweise zur Messung der Sauerstoffkonzentrationen in Transportverpackungen verwendet [3].
Um die prinzipbedingten Vorteile der lumineszenzbasierten Gassensorik für kontinuierliche Messaufgaben zu nutzen, gilt es, neue langzeitstabile und zugleich gasempfindliche Luminophore zu finden.

Eine interessante Alternative ist halbleitendes, photostabiles Zinkoxid. Dieses weist bei entsprechender kristalliner Beschaffenheit ebenfalls Lumineszenzemissionen auf, welche von der umgebenden Gasatmosphäre beeinflusst werden [4]. Im Gegensatz zu den zuvor beschriebenen Metallkomplexen ist der Mechanismus des Lumineszenz-Quenchings für die Metalloxide jedoch nicht abschließend geklärt. Optimierungen der Materialsynthese und der Signalauswertung können deshalb bisher nur rein empirisch vorgenommen werden.

Ziel dieser Arbeit ist es, ausgehend von bekannten Mechanismen aus dem Bereich der resistiven Gassensorik mit Metalloxiden, Hinweise auf den Quenching-Mechanismus zu gewinnen. Bei resistiven Sensoren wird üblicherweise der Leitwert von thermisch aktiviertem Zinkoxid als Sensorgröße verwendet. Durch simultane Messungen von Lumineszenz und Leitwert an Zinkoxid-Partikeln sollen bei variierender Sauerstoffkonzentration Indizien für die Quenching-Mechanismen gefunden werden. 


\section{Zinkoxid}

Zinkoxid $(\mathrm{ZnO})$ ist ein halbleitendes Metalloxid mit einer Bandlücke von ca. $3,2 \mathrm{eV}$. Bei hochenergetischer optischer Anregung, z.B. mit einer Wellenlänge von $280 \mathrm{~nm}(4,43 \mathrm{eV})$, zeigt Zinkoxid ausgeprägte Lumineszenzemissionen. Ursache dafür sind strahlende Übergänge der angeregten Elektronen vom Leitungs- in das Valenzband (Abbildung 1). Direkte Relaxation der Elektronen führt zur Emission mit einer Wellenlänge von ca. $384 \mathrm{~nm}$ (3,2 eV), dem Bandabstand von $\mathrm{ZnO}$ (Band-Band-Lumineszenz). Je nach Dotierung bzw. Defekten kommt es zudem zur indirekten Relaxation über Defektniveaus, welche im gezeigten Beispiel zu einer breiten Emissionsbande bei ca. $510 \mathrm{~nm}(2,43 \mathrm{eV})$ führt (Defekt-Lumineszenz). Der Anteil der BandBand- und Defekt-Lumineszenz hängt dabei von der Kristallinität des verwendeten Zinkoxids ab [5].

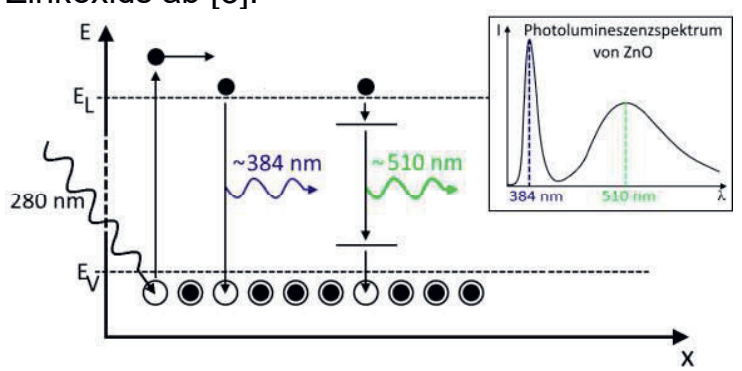

Abb. 1: Schematische Darstellung der Energieniveaus von Zinkoxid. Links: UV-Anregung bei $280 \mathrm{~nm}$. Mitte: Band-Band-Lumineszenz bei ca. $384 \mathrm{~nm}$. Rechts: Defekt-Lumineszenz bei ca. $510 \mathrm{~nm}$. Einschub: Charakteristisches Photolumineszenzspektrum von $\mathrm{ZnO}$.

Neben der Lumineszenz, kann zudem die Leitfähigkeit des Zinkoxids optisch aktiviert werden. Die Elektronen können dabei, je nach Energie, aus leitungsbandnahen Fehlstellen oder aus dem Valenzband in das Leitungsband gehoben werden und dort zur Leitfähigkeit beitragen.

\section{Experimentelles}

\subsection{Messzelle}

Um simultan den Leitwert und die Lumineszenz der Zinkoxid-Partikel bei variierenden Gaskonzentrationen zu erfassen, wurde eine Messzelle entworfen. Diese besteht aus einer Standard-Fluoreszenzküvette und einem Küvetteneinsatz (Abbildung 2). Der Küvetteneinsatz schließt die Küvette gasdicht $a b$ und fixiert eine Keramikplatine, die als Sensorsubstrat dient. Auf der Keramikplatine ist eine Interdigital-Elektrodenstruktur aus
Kupfer aufgebracht, die mit einer Schicht aus Chemisch Gold (ENIG) passiviert ist. Der Elektrodenabstand und die Elektrodenbreite betragen $100 \mu \mathrm{m}$. Durch den Küvetteneinsatz können die Drähte zur Kontaktierung der Interdigitalstruktur nach außen geführt und die Messzelle mit Prüfgasen beaufschlagt werden. Die Messzelle ermöglicht es, die Probe optisch anzuregen und unter einem Winkel von $90^{\circ}$ zur Anregung die Lumineszenzemission zu beobachten. Zeitgleich kann der Leitwert der Probe erfasst und die Gaskonzentration innerhalb der Messzelle variiert werden.

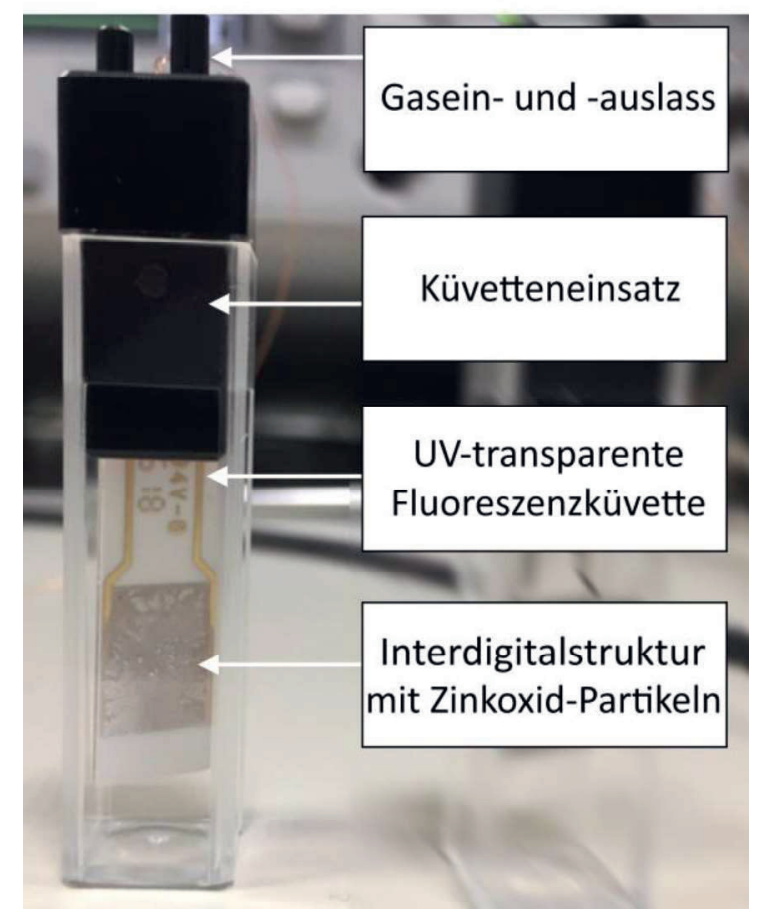

Abb. 2: Verwendete Messzelle, bestehend aus einer UV-transparenten Laborküvette, dem Küvetteneinsatz sowie der Keramikplatine mit ZinkoxidPartikeln.

\subsection{Sensorpräparation}

Für die Messungen in dieser Arbeit wurden kommerziell verfügbare Zinkoxid-Nanopartikel verwendet (abcr.de - Art.-Nr.: AB354609). Zur Präparation wurden $50 \mathrm{mg}$ der Zinkoxid-Partikel und $2 \mathrm{ml}$ Ethanol in ein Schnappdeckelglas gegeben und in einem Ultraschallbad für $5 \mathrm{~min}$ dispergiert. Die Keramikplatinen wurden auf einer Labor-Heizplatte auf $60^{\circ} \mathrm{C}$ erwärmt. Anschließend wurden mit einer Mikroliterpipette $20 \mu \mathrm{l}$ der Dispersion entnommen und in einem Zug auf die Interdigitalstruktur getropft. Nachdem das Ethanol verdampft war, wurde dieser Vorgang zwei weitere Male wiederholt. Das Substrat wurde anschließend mit zwei Kupferlackdrähten kontaktiert und in der Messzelle montiert. 


\subsection{Messaufbau}

Abbildung 3 zeigt schematisch die Anordnung der elektrischen und optischen Komponenten des Messaufbaus. Das Zinkoxid wird über eine Leuchtdiode mit einem Emissionsmaximum bei $280 \mathrm{~nm}$ angeregt, welche mit einer Konstantstromquelle betrieben wird. Die optische Leistung beträgt ca. $1 \mathrm{~mW}$. Die Lumineszenzemission wird über eine Kollimatorlinse erfasst und über eine optische Faser an ein Faserspektrometer geleitet (Ocean Optics, Typ: Flame S). Um die reflektierten Anteile der intensiven anregenden Strahlung zu filtern, ist vor der Kollimatorlinse ein optischer Langpassfilter mit einer Grenzwellenlänge von $375 \mathrm{~nm}$ angebracht.



Abb. 3: Schematische Darstellung des Messaufbaus.

Um den Leitwert unter definierten Bedingungen zu messen, wird die Interdigitalstruktur in Reihe zu einem $1 \mathrm{M} \Omega$ Widerstand an einem Labornetzteil mit einer konstanten Ausgangsspannung von $20 \mathrm{~V}$ betrieben. Der Leitwert der Zinkoxid-Partikel wird anschließend aus der gemessenen Spannung über der Interdigitalstruktur berechnet (Multimeter: HP 34401A). Die Beaufschlagung mit Gas erfolgt über eine PC-gesteuerte Gasmischanlage. Die Ausgangsgase sind Stickstoff (Fa. Linde, Reinheit 5.0), sowie synthetische Luft (Fa. Linde, $20 \%$ Sauerstoff, KWfrei). Um den Einfall von Umgebungslicht zu vermeiden, wurden die optischen Komponenten in einem Aluminiumblock angeordnet. Abbildung 4 zeigt den Messaufbau mit eingesetzter Messzelle.

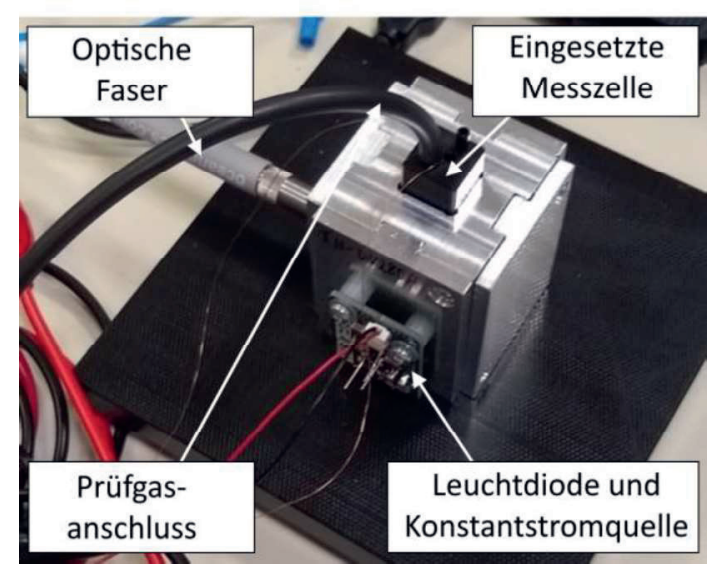

Abb. 4: Messaufbau.

\subsection{Messdatenerfassung}

Um thermisch stabile Bedingungen $\mathrm{zu}$ schaffen, wurde die Leuchtdiode zwei Stunden vor den eigentlichen Messungen in Betrieb genommen und die Messzelle während dieser Zeit mit Stickstoff gespült. PC-gesteuert wurde die Sauerstoffkonzentration in der Messzelle anschließend schrittweise erhöht (60 Minuten je Konzentrationsänderung). Der Volumenstrom beträgt dabei $100 \mathrm{ml} / \mathrm{min}$. Der Spannungsabfall über der Zinkoxidprobe, sowie das optische Spektrum wurden über eine PC-Schnittstelle erfasst. Aus dem Spannungsabfall lässt sich anschließend der Leitwert der Probe berechnet. Die Messdaten des Spektrometers wurden auf die maximale Lumineszenzintensität normiert. Eine Korrektur der Lumineszenzspektren, um beispielsweise systembedingte Messartefakte zu kompensieren, erfolgte nicht.

\section{Messergebnisse und Diskussion}

Im ersten Schritt soll gezeigt werden, dass das verwendete Zinkoxid ein gasabhängiges Lumineszenz-Verhalten aufweist. Dazu wurden mit dem zuvor beschriebenen

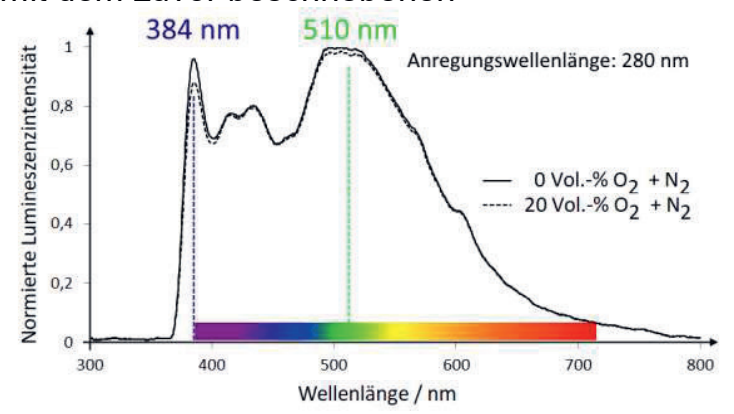

Abb. 5: Photolumineszenzspektrum von Zinkoxid bei Abwesenheit (durchgezogene Linie) und bei Anwesenheit (gestrichelte Linie) von Sauerstoff. 
Aufbau Lumineszenzspektren unter unterschiedlichen Gasatmosphären aufgenommen (Abbildung 5). Die Spektren weisen die für Zinkoxid charakteristischen Band-Band-Lumineszenzen bei $384 \mathrm{~nm}$, sowie die defektbedingten Lumineszenzen bei ca. $510 \mathrm{~nm}$ auf.

Bei Variation der Gasatmosphäre kann eine Veränderung der Lumineszenzintensität festgestellt werden. In Anwesenheit von Sauerstoff nimmt die Lumineszenzintensität ab (Lumineszenz-Quenching). Bei einer Variation der Sauerstoffkonzentration von 0 auf $20 \mathrm{Vol} .-\%$ wird ein Rückgang der ZnO-Lumineszenzintensität $(384 \mathrm{~nm})$ von ca. $10 \%$ beobachtet (Abbildung 6). Der Einfluss des Sauerstoffs auf die Defekt-Lumineszenz $(510 \mathrm{~nm})$ ist mit einer Abschwächung von ca. $2 \%$ wesentlich geringer.

Die Nebenmaxima bei $410 \mathrm{~nm}$ und $430 \mathrm{~nm}$ sind Messartefakte, die auf die Komponenten des Messaufbaus zurückzuführen sind und zeigen dementsprechend kein gasabhängiges Verhalten. So sind den gemessenen Spektren zum einen, trotz des eingesetzten UV-Filters, Anteile des Spektrums der UV-Leuchtdiode überlagert; diese emittiert geringe Anteile im sichtbaren Spektralbereich. Zum anderen weisen alle optischen Komponenten (Kollimatorlinse, opt. Faser, Detektor) ein wellenlängenabhängiges Übertragungsverhalten auf, sodass der tatsächliche Anteil der Band-Band- und Defekt-Lumineszenz ggfs. anders gewichtet ist.

Ein Vergleich des zeitlichen Verlaufs der Intensität der Band-Band-Lumineszenz $(384 \mathrm{~nm})$ mit dem Verlauf des simultan gemessenen Leitwerts bei variierender Sauerstoffkonzentration zeigt eine starke Korrespondenz der beiden Signale (Abbildung 6).

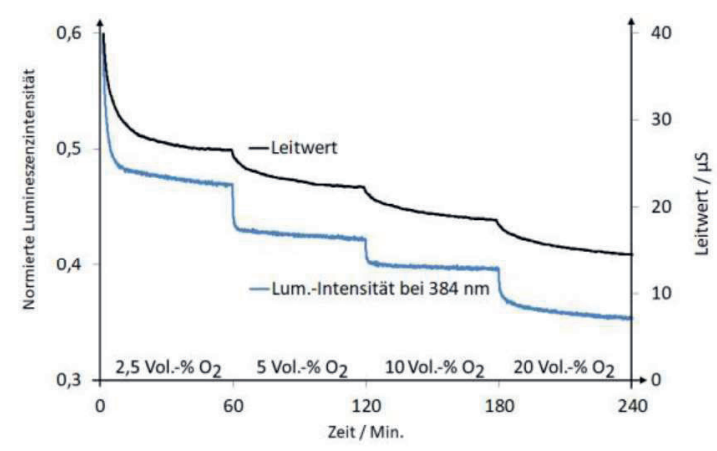

Abb. 6: Zeitliche Änderung der Lumineszenzintensität $(384 \mathrm{~nm})$ und des Leitwerts bei Variation der Sauerstoffkonzentration.

Sowohl die Lumineszenzintensität als auch der Leitwert nehmen mit steigender Sauerstoff- konzentration ab. Die Abnahme des Leitwertsignals erfolgt durch die Lokalisierung freier Elektronen an der Oberfläche der $\mathrm{ZnO}$ Partikel.

Zusätzlich zur Bandverbiegung an den Korngrenzen trägt auch die Verringerung der freien Ladungsträger zur Abnahme des Leitwertes bei (Abbildung 7). Die beobachtete Korrespondenz der beiden Signale legt nahe, dass diese Abnahme der freien Ladungsträger auch die Ursache für das LumineszenzQuenching darstellt. Die optisch angeregten Elektronen werden durch Sauerstoff-Adsorbate an der Grenzfläche der ZnO-Partikel lokalisiert und können nicht strahlend relaxieren.

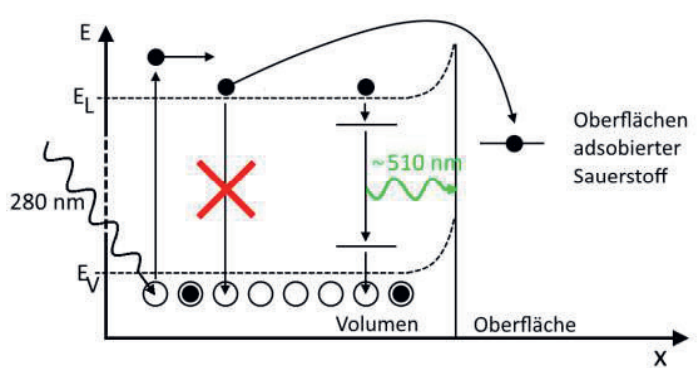

Abb. 7: Schematische Darstellung des Einflusses von Sauerstoff auf der Partikeloberfläche auf die Lumineszenzemission.

Somit ist davon auszugehen, dass Zinkoxid als Luminophor ähnliche gassensitive Eigenschaften wie im Fall der resistiven Signalauswertung aufweisen wird. Bemerkenswert ist jedoch, dass sich die Dynamik der Signale bei Variation der Gaskonzentration deutlich unterscheidet.

Die Lumineszenzintensität weist eine steilere Flanke auf und stabilisiert sich schneller als das Leitwertsignal. Die Ursache dafür liegt wahrscheinlich in der Überlagerung parasitärer Effekte durch Partikel-Partikel-Übergänge im Zinkoxid-Pulver sowie in Umladungseffekten auf der Oberfläche. Die damit verbundenen Variationen in den Raumladungsschichten bzw. den Bandverbiegungen an den Korngrenzen beeinflussen das Leitwertsignal stark. Der beschriebene Mechanismus zum Lumineszenz-Quenching jedoch ist in gewissen Grenzen unabhängig von der Verbiegung der Bänder und reagiert primär auf die Zahl der freien Elektronen. Dieser Ansatz eröffnet somit eine Möglichkeit zur Signalstabilisierung.

\section{Zusammenfassung und Ausblick}

In dieser Arbeit wurde gezeigt, dass die Lumineszenz von Zinkoxid-Partikeln grundsätzlich zur Detektion von Sauerstoff genutzt werden 
kann und es wird ein Quenching-Mechanismus für Zinkoxid vorgeschlagen. Die beobachtete Korrespondenz des Lumineszenzsignals mit dem Leitwert legt nahe, dass das Quenching auf der Lokalisierung von Leitungselektronen durch Adsorbate an der Oberfläche der Zinkoxidpartikel beruht. Damit unterscheidet sich der Mechanismus qualitativ vom Quenching der Metall-Komplexe; eine inhärente Selektivität bzgl. des Sauerstoffs ist im Fall des Zinkoxids nicht zu erwarten. Das Lumineszenzsignal zeigt allerdings ein deutlich schnelleres Ansprechverhalten als das Leitwertsignal. Weitere Studien müssen zeigen, wie diese Dynamik von den Materialparametern, wie z.B. der Kristallitgröße des Zinkoxids, beeinflusst wird.

\section{Literaturnachweis}

[1] X. Wang, O. Wolfbeis, Chem. Soc. Rev., 36663761 (2014); doi: 10.1039/C4CS00039K

[2] O. Wolfbeis, Bioessays. 37(8):921-8 (2015); doi: 10.1002/bies.201500002

[3] P. Hartmann, M. Leiner, P. Kohlbacher, Sensors and Actuators B: Chemical. 51. 196202; doi: 10.1016/S0925-4005(98)00188-9

[4] V. M. Zhyrovetsky, D.I. Popovych, S. S. Savka, A. S. Serednytski, Nanoscale Research Letters 12:132 (2017) doi: 10.1186/s11671-017-1891-5

[5] O. Marin, M. Tirado, N. Budini, E. Mosquera, C. Figueroa, D. Comedi, Materials Science in Semiconductor Processing, 56, 59-65 (2016); doi: 10.1016/j.mssp.2016.07.007 